\title{
Tabak, die am weitesten verbreitete Giftpflanze
}

\author{
Till KaSieLKe \& ARMin JAGeL
}

\begin{abstract}
Tobacco (Nicotiana) is poisenous plant of the year 2009. Nicotiana tabacum and N. rustica are the economical most important species. Their biology, cultivation, use of the leaves and toxicology are described. Some Nicotiana species and cultivars are ornamental plants in our gardens.
\end{abstract}

\section{Zusammenfassung}

Tabak (Nicotiana) ist Giftpflanze des Jahres 2009. Nicotiana tabacum und N. rustica sind die beiden wirtschaftlich wichtigsten Arten. Ihre Biologie, Kultur, Verwendung der Blätter und Giftigkeit werden beschrieben. Einige Tabak-Arten und Kultivare werden in unseren Gärten als Zierpflanzen verwendet.

\section{Einleitung}

Die Gattung Nicotiana umfasst 75 Arten (HAMMER 2000). Von globaler wirtschaftlicher Bedeutung sind aber nur zwei Arten, nämlich der Virginische (Nicotiana tabacum) und der BauernTabak (N. rustica); von diesen beiden überwiegt die Bedeutung des ersten (Abb. 1) bei weitem. Die Verwendung zum Rauchen, Schnupfen und Kauen macht ihn neben Alkohol zur weltweit mengenmäßig am meisten konsumierten Genussdroge. In S- und M-Amerika spielen einige weitere Arten eine entsprechende, aber nur lokale Rolle. In Deutschland wird der Anbau von Tabak zunehmend reduziert und dürfte in den nächsten Jahren aufgrund auslaufender Subventionen ganz zum Erliegen kommen. Neben den oben genannten Arten, die bei uns gelegentlich auch als sogenannte Vagabunden verwildern (sie verschwinden bald wieder und treten dann an anderer Stelle erneut kurzfristig auf), werden als einjährige Sommerblumen einige weitere Arten, Hybriden und Sorten gepflanzt.

Für das Jahr 2009 wurde Tabak vom Botanischen Sondergarten Wandsbek (Hamburg) zur Giftpflanze des Jahres gekürt. Mit der Nennung einer solchen Pflanze will der Garten seit mittlerweile 5 Jahren auf giftige Pflanzen aufmerksam machen, die in Haus und Garten wachsen und deren Giftigkeit in der Öffentlichkeit nicht genügend bekannt ist. Aus unserer Sicht ist die Wahl von Tabak dabei etwas unglücklich, weil es in Deutschland wohl kaum zu Vergiftungen durch die (lebende) Tabakpflanze kommt, weder von den feldmäßig angebauten Arten noch den Zierpflanzen. Der Grund hierfür ist einfach, denn die Pflanze verführt nicht z. B. durch attraktive Früchte zum Verzehr. Anders als die anderen „Jahrespflanzen“ (Blume oder Baum des Jahres, etc.) wird die Giftpflanze des Jahres nicht von einer Gruppe Spezialisten ernannt, sondern durch Abstimmung zwischen vier Kandidaten ausgewählt, wobei jeder Interessierte teilnehmen kann. So hat in diesem Jahr der Tabak die bei uns als Giftpflanze deutlich bedeutsameren Arten Aronstab, Efeu und Lebensbaum besiegt. Die Wahl dürfte sicherlich durch die anhaltenden Diskussionen um das Rauchverbot im letzten Jahr beeinflusst worden sein und somit eher eine politisch-pädagogische Entscheidung darstellen. Die Tabakpflanze ist aber nicht nur eine Giftpflanze, sondern wurde lange auch als Heilpflanze verwendet. Wie bei fast allen Giftpflanzen ist der Übergang zur Heilpflanze fließend und die Wirkung hängt von der Dosis ab. Die Besonderheit beim Tabak ist, dass jeder Raucher täglich eine Anzahl Zigaretten raucht, die ihn anders konsumiert - sofort töten würde.

\section{Vorkommen und äußeres \\ Erscheinungsbild}

Der häufigste als Genussdroge angebaute Tabak ist Nicotiana tabacum (Abb. 1), eine einjährige, bis $3 \mathrm{~m}$ hohe Pflanze mit rötlichen Blüten, die eine lange Kronröhre aufweisen. Sie hat einen aufrechten Spross mit 20 bis 30 Blättern, wenigen Seitentrieben und einen endständigen Blütenstand, der zwischen 100 und 150 Blüten bilden kann. Diese bringen bei Fruchtreife bis zu 1 Mio. Samen pro Pflanze hervor. Die zweite Tabak-Art, die als Genusspflanze eine größere 
Rolle spielt, ist der Bauern-Tabak (N. rustica). Er bleibt mit bis zu 1,5 m Höhe kleiner und hat gelblich grüne Blüten (Abb. 2, 3).

Beide Arten wurden schon vor der Entdeckung Amerikas von den Einheimischen in Süd- und Mittelamerika angebaut, Wildvorkommen sind nicht bekannt. Nicotiana tabacum ist wahrscheinlich eine Hybride aus $N$. sylvestris (aus Nord-Argentinien) und $N$. tomentosiformis (Bolivien). Nicotiana rustica dürfte in Peru als Hybride aus $N$. undulata und $N$. paniculata (Abb. 4) entstanden sein, die heute noch an den Westhängen der Anden vorkommen.

\section{Geschichte des Tabaks und des Rauchens}

Als Kolumbus 1492 in Kuba an Land ging, war Tabak dort bereits ein allgemein bekanntes Genussmittel. GONZALES DE OVIEDO Y VALDEZ, ein Freund des Amerika-Entdeckers, berichtet über das schädliche Laster der Indianer, die das Kraut in einer Pfeife rauchten, bis sie bewusstlos wurden und wie berauscht auf die Erde hingestreckt in einen tiefen Schlaf verfielen (SCHMidTBAUER \& SCHEIDT 1999). Andere Völker rauchten ein in ein Maisblatt eingewickeltes Kraut, was sie „zikar" oder "tabacos" nannten (Wolters 1994). Schon früher war der Tabak bei den Azteken und Tolteken ein heiliges Kraut. Die angehenden Medizinmänner in Peru, Ekuador und Guyana mussten bei ihrem Initiationsritual Tabakwasser trinken, um ihre Tauglichkeit unter Beweis zu stellen, was in Anbetracht der Giftigkeit oral aufgenommenen Nikotins eine sehr gefährliche Angelegenheit darstellte. Die Novizen erfuhren hierbei halluzinatorische Zustände, Bewusstlosigkeit und - wenn sie Pech hatten, also „ungeeignet“ waren - einen tödlichen Kollaps (SCHMidTBAuer \& SCHeidT 1999).

Um 1560 kam Tabak nach Europa, wo er zunächst als Zierpflanze verwendet wurde. Durch den französischen Botschafter in Lissabon, JEAN Nicot DE VILLEMAIN, wurde der Tabak verbrei-

Abb. 1 (oben): Virginischer Tabak (Nicotiana tabacum) blühend in einem Schaugarten.

Abb. 2 (Mitte): Bauern-Tabak (Nicotiana rustica).

Abb. 3 (unten): Blüte von Nicotiana rustica.
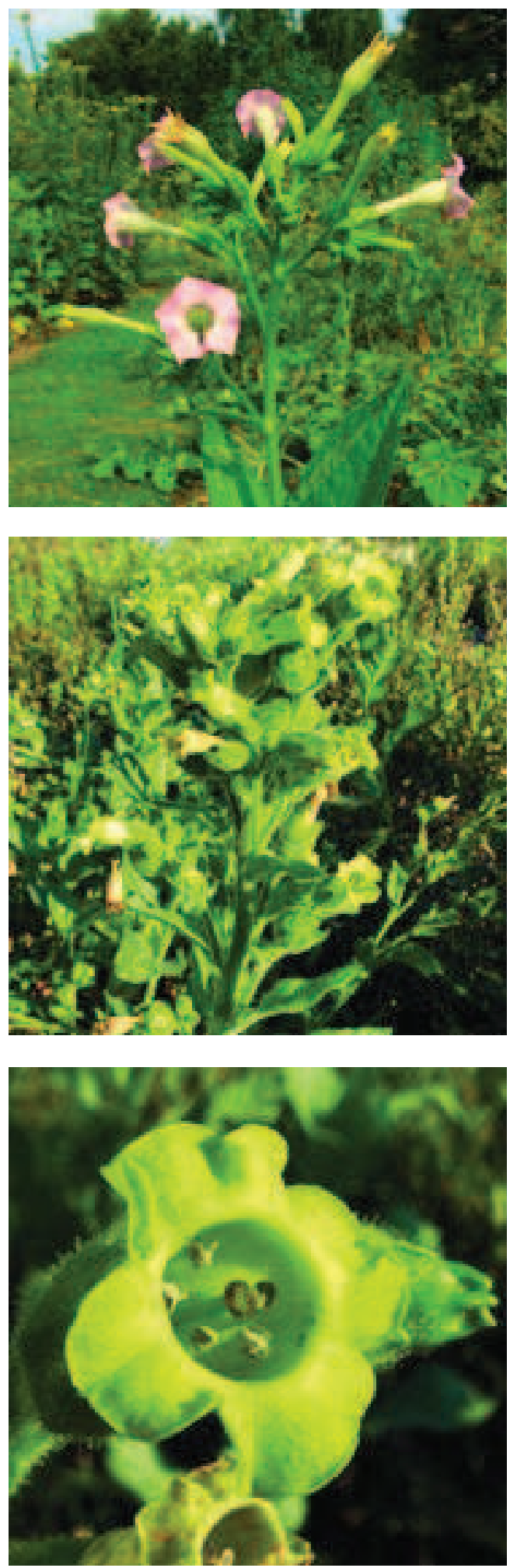

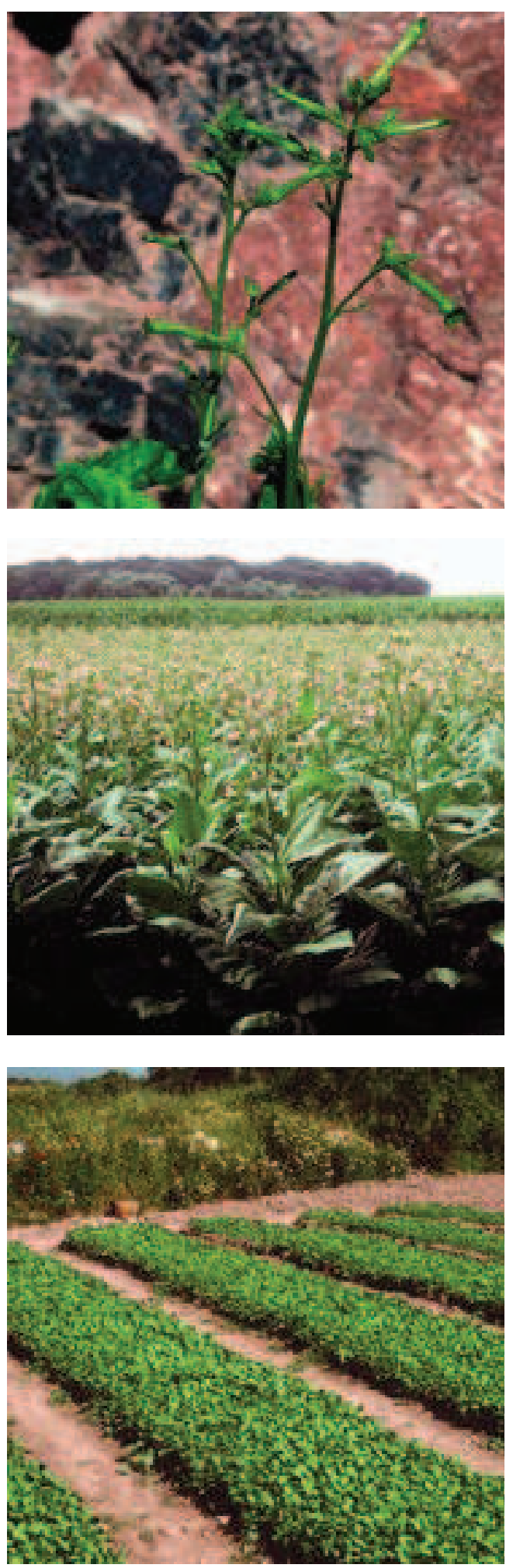

tet; nach ihm wurde später auch die Gattung Nicotiana benannt. Die Aufnahme der als heidnisch angesehenen Genussdroge im Europa der anbrechenden Renaissance war zunächst zwiespältig. Als der Seefahrer und Begleiter von Kolumbus, RODRIGO DE JEREZ, rauchend in seiner Heimatstadt über die Straße ging, hielten ihn die Bürger für gotteslästerlich, vom Teufel besessen und lieferten ihn der Inquisition aus. Sie kerkerten ihn ein und ließen ihn erst wieder frei, als sich der Tabakgenuss in Spanien allmählich durchgesetzt hatte. Auch im Lüneburgischen drohte man noch 1691 den Untertanen, die sich dem „liderlichen Werk des Tabaktrinkens" hingaben, mit der Todesstrafe. Die Bürger von Berlin ließen 1848 während der März-Revolution erst von ihrem aufständischen Begehren ab, als man ihnen das Recht zugestand, auf der Straße zu rauchen (SCHMidTbaUer \& SCHEIDT 1999). Nachdem zunächst das Pfeiferauchen und das Schnupfen von Tabak üblich waren, setzte sich seit Beginn des 19. Jh. das Zigarrerauchen durch. Erst in den 1860er Jahren kam in der Türkei die Zigarette auf (HAMMER 2000).

Im Jahre 1611 wurde in Spanien Tabak als Geldquelle erkannt, indem man zunächst eine Steuer auf den Import erließ und 1632 dann auch auf den Verkauf (WOLTERS 1994). Noch heute wird immer wieder die Tabaksteuer angehoben, wenn Finanzlöcher zu stopfen sind; allerdings gibt man heute vor, aus Gründen der staatlichen Verantwortung für die Gesundheit dazu verpflichtet zu sein. Um jedoch nicht allzu viele Raucher dabei zu verlieren (und den erhofften finanziellen Segen dadurch zu verpassen), erfolgen solche Steueranhebungen in der Regel in kleinen Schritten.

\section{Anbau}

Die Hauptproduktion des Tabaks liegt heute in China, größtes Produktionsland im Mittelmeergebiet ist die Türkei, nennenswerter Anbau in

Abb. 4 (oben): Nicotiana paniculata, eine der vermutlichen Ausgangsarten des Bauern-Tabaks (Nicotiana rustica).

Abb. 5 (Mitte): Tabakfeld am Oberrhein.

Abb. 6 (unten): Anzuchtbeete (Milet, Westtürkei). 
Europa wird in Italien betrieben (LIEBEREI \& REISDORFF 2007). Da Tabak eine sehr wärmebedürftige Art ist, findet man günstige Anbaubedingungen unter tropischen und subtropischen Bedingungen, aber auch in gemäßigten Breiten wird Tabakanbau betrieben. In Deutschland lohnt sich das besonders im wärmebegünstigten Südwesten (Abb. 5). Unter mitteleuropäischen Bedingungen muss eine besondere Anbautechnik angewandt werden. So erfolgt hier die Aussaat unter Glas im Warmbeet und die Setzlinge werden erst zu Beginn der warmen Jahreszeit Ende Mai ins Freiland ausgepflanzt. Zum optimalen Wachstum und zur Reife benötigt Tabak Temperaturen zwischen 25 und $35^{\circ} \mathrm{C}$ (LIEBEREI \& REISDORFF 2007).

Der Tabakanbau wird heute in der EU subventioniert, 150 Mio. Euro gehen jedes Jahr an Deutschland. Seit 2005 werden allerdings 20\% davon dafür verwendet, die Tabakbauern zum Umsteigen auf andere Kulturpflanzen zu bewegen, 2010 läuft die Subventionierung ganz aus. Nachdem der Tabakanbau in Deutschland seit den 1950er Jahren bis heute auf ein Zehntel zurückgegangen ist, dürfte dies vermutlich das Aus bedeuten, wie dies in Österreich schon der Fall ist. In wärmeren Ländern, wie z. B. in der Türkei, werden die Jungpflanzen ebenfalls zunächst in kleinen Parzellen im Freiland vorgezogen, da die notwendige Bewässerung so einfacher und sparsamer erfolgen kann (Abb. 6, 7). Auf künstlich erzeugte Bodenwärme oder Schutz durch Glas kann hier verzichtet werden. Um den Nikotingehalt in den Blättern zu steigern, werden während des Wachstums oftmals die Blüten entfernt und die Seitentriebe der Pflanze während des Wachstums frühzeitig herausgebrochen. Die Erntezeit beginnt mit der Vergilbung der Blätter, die an der Pflanze von unten nach oben erfolgt. Nur für die Erzeugung von Zigarrentabak werden die Blätter schon in

Abb. 7 (oben): Tabak-Jungpflanzen (Milet, Westtürkei).

Abb. 8 (Mitte): Junge Setzlinge wurden an Ort und Stelle in Reihen gepflanzt (Milet, Westtürkei).

Abb. 9 (unten): Stehen gebliebene Tabak-Pflanze am Rande eines abgeernteten Tabakfeldes (Milet, Westtürkei).
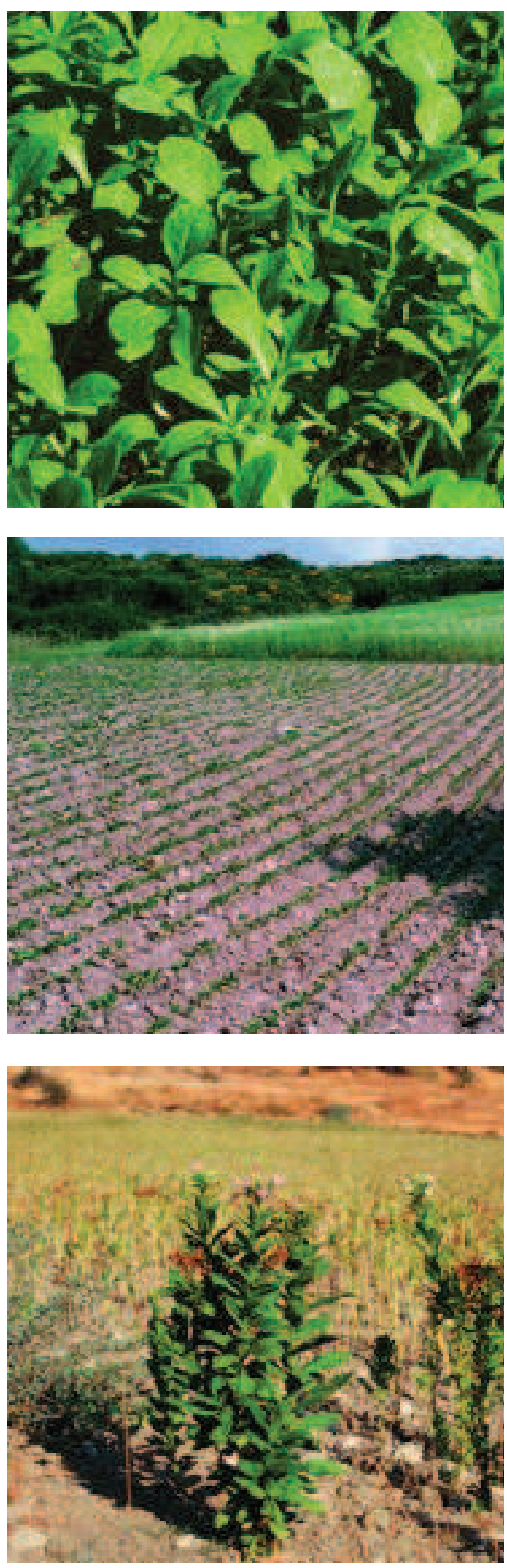

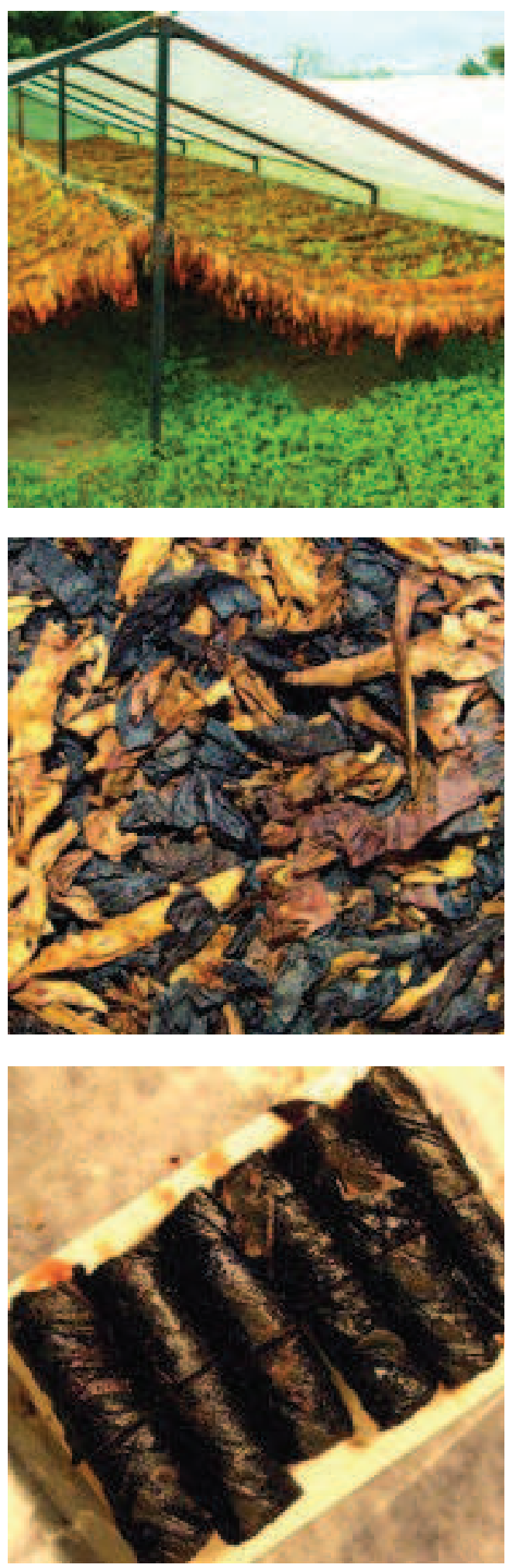

grünem Zustand gepflückt. Eine optimale Ernte geschieht mit der Hand, indem die Blätter der Reihe nach, dem Reifezustand entsprechend, gepflückt werden. Die Blätter aus den verschiedenen Spross-Zonen haben unterschiedliche Bezeichnungen und Verwendungen, z. B. werden die dritten und vierten Blätter (Sandblätter) gerne als Deckblätter für Zigarren verwendet, die mittleren Blätter liefern den besten Tabak. In rationalisierten Betrieben Amerikas (d.h. Pflücker wurden wegrationalisiert) erfolgt die Ernte aller Blätter (mit Ausnahme der Sandblätter) zeitgleich maschinell.

Nach der Ernte und der Entfernung der Mittelrippe werden die Blätter zunächst getrocknet. Dies erfolgt entweder traditionell (wie zumeist in den Mittelmeerländern) durch 1-2-monatige Lufttrocknung. Die Blätter dürfen dabei nicht zu schnell trocknen, um wichtige Reifungs- und Abbauprozesse in den Blättern zu ermöglichen (Abb. 10). Daneben wurde eine Heißlufttrocknung entwickelt, wobei das Verfahren durch einen kontrollierten Trocknungsprozess auf eine Woche verkürzt werden kann. Nach der Trocknung werden die Blätter gelagert und hierbei durch Fermentierung braungelb. Eiweiße, Zucker und Stärke werden dabei abgebaut und es entwickeln sich Aromastoffe, die den typischen Geschmack erzeugen. Das Produkt kommt dann als Tabak auf den Markt, wobei es für die unterschiedliche Verwendung verschiedene Sorten und Ernten gibt: Zigaretten-, Zigarren- und Pfeifentabak sowie Schnupfund Kautabak (Abb. 11-14).

\section{Tabak als Heilpflanze}

Schon Ende des 16. Jh. wurde Tabak aufgrund der Wirkung des Nikotins in Europa als Arzneimittel eingesetzt, die frühe Verwendung wird in der Encyklopädie der Volksmedizin von FRIEDRICH MosT (1843) beschrieben. Von den Maya

Abb. 10 (oben): Lufttrocknung der geernteten Tabakblätter in Makedonien (Griechenland).

Abb. 11 (Mitte): Pfeifentabak.

Abb. 12 (unten): Kautabak. 
übernahm man die Verwendung als Einlauf bei Koliken, Verstopfung sowie bei „krampfhafter Darmeinklemmung, schmerzhafter Gonorrhöe und Blasenkrampf“. Schnupftabak wurde gegen Migräne empfohlen und ,ist, rein und gut bereitet, auch als Hausmittel gegen Stockschnupfen, rheumatische Kopf- und Zahnschmerzen, gegen schwache, blöde, triefende Augen etc. als heilsam bekannt" (zit. nach WOLTERS 1994). Neben der Wirksamkeit gegen einige weitere Krankheiten wird hier sogar darauf hingewiesen, dass zu viel Rauchen und Schnupfen von Tabak die Zeugungskraft abstumpft, und es wird sehr verliebten Personen als Antiaphrodisiacum empfohlen (Wolters 1994). Noch heute soll Nikotin in China bei Wurmerkrankungen angewandt werden. Bei uns ist die arzneiliche Verwendung gegen die genannten Krankheiten heute aufgrund neuerer Medikamente nicht mehr in Gebrauch. Nikotin wird allerdings noch in Form von Pflastern oder Kaugummis unterstützend bei der Rauchentwöhnung eingesetzt. In der Homöopathie wird Nikotin z. B. bei Erkrankungen der Augen, des arteriellen Gefäßsystems sowie des Magen-Darm-Traktes verwendet.

\section{Giftstoffe}

Nikotin und Teer sind keineswegs die einzigen Giftstoffe, die der Raucher mit jedem Zug aufnimmt. Inzwischen sind weit über 1500 chemische Verbindungen im Tabak und Tabakrauch festgestellt worden, darunter Schwermetalle wie das hochgiftige Cadmium sowie die nicht minder gefährlichen Schadstoffe Blausäure, Ammoniak und Arsen. Das im Rauch enthaltene Formaldehyd sorgt für das Brennen in den Augen. In der ganzen Pflanze sind die PyridinAlkaloide Nicotin, Nornicotin, Myosmin, Isonicotin, Anabasin, Anatabin und weitere Nebenalkaloide enthalten. Der Hauptwirkstoff ist das

Abb. 13 (oben): Zigarettentabak zum Selbstdrehen mit Blättchen.

Abb. 14 (Mitte): Schnupftabak, wird gerne von Kumpeln im Bergbau genutzt, weil das Rauchen unter Tage verboten ist. Abb. 15 (unten): Ziertabak.
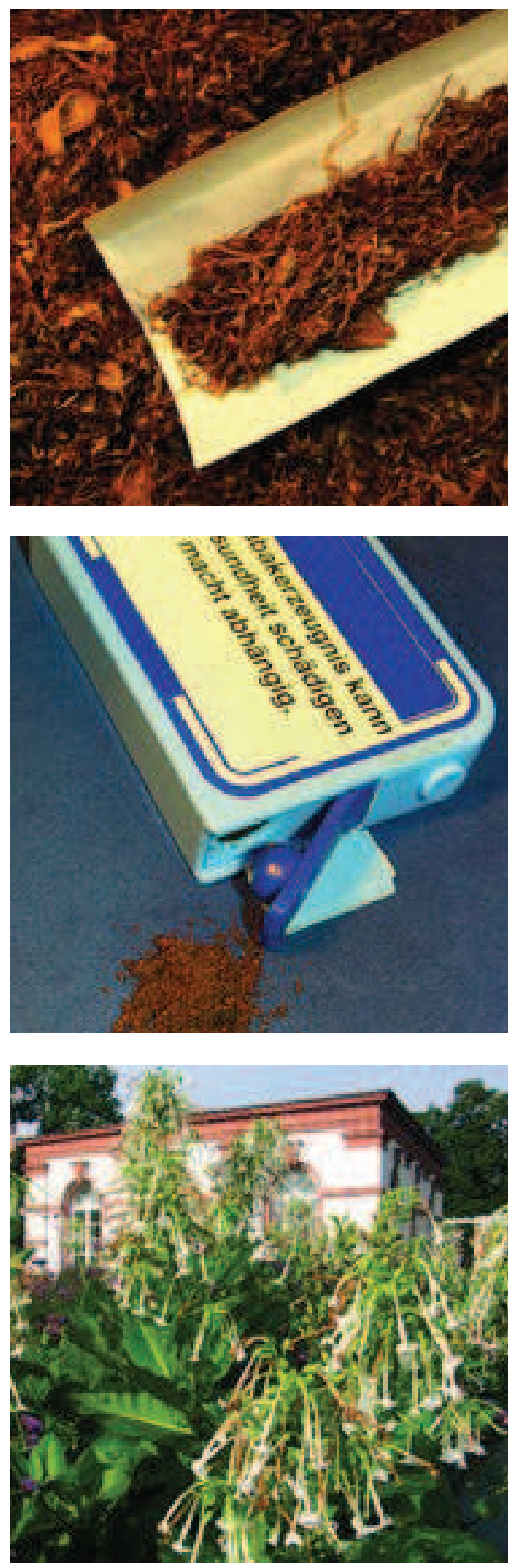
Alkaloid Nikotin, das im Organismus einerseits eine Kontraktion der Blutkapillaren, Schweißausbrüche sowie Absinken der Hauttemperatur (bis auf $7^{\circ} \mathrm{C}$ !) bewirkt, gleichzeitig aber über Adrenalin den Kreislauf anregt.

Nikotin entsteht erst während des Wachstums der Pflanzen in der Wurzel, von dort wird es nach oben transportiert und reichert sich allmählich in Sprossachse und Blättern an. Daher ist die ganze Pflanze, mit Ausnahme der Samen, stark giftig. Der Nikotingehalt in den Blättern des Virginischen Tabaks schwankt zwischen 0,05 und $6 \%$. Der Bauerntabak enthält sogar bis zu $7,5 \%$ Nikotin. Aus ihm wird der starke russische Machorka hergestellt. Im Blaugrünen Tabak (Baumtabak, Strauchtabak, N. glauca) konnte kein Nikotin nachgewiesen werden. Dagegen sind Nornikotin und Anabasin sowohl in Blättern als auch in den Blüten zu gleichen Anteilen enthalten. In Mexiko wird auch N. glauca als Tabak geraucht. Er soll sehr stark sein und wird Marihuana, gelegentlich auch Macuchi genannt. Mit dem Cannabis-Produkt hat diese Art Mari- huana natürlich nichts zu tun (RоTH et al. 2008).

Die tödliche Dosis an Nikotin wird bei Erwachsenen nach oraler Applikation auf 40-60 mg geschätzt. Es wird auch eine tödliche Dosis von $1 \mathrm{mg} / \mathrm{kg}$ Körpergewicht angegeben (HILLER \& MenZig 2003). Man kann davon ausgehen, dass in einer Zigarette ca. $10 \mathrm{mg}$ und in einer $\mathrm{Zi}$ garre ca. $90 \mathrm{mg}$ Nikotin enthalten sind. Dies bedeutet, dass 5 Zigaretten oder eine halbe Zigarre eine tödliche Menge darstellen. Eine Gefahr stellen in Wasser aufgelöste Reste von Rauchwaren dar, da sich das Nikotin in Wasser gut löst. Konzentrierte Nikotinlösungen führen innerhalb von Minuten zum Tod. Nach sehr großen Mengen, die vor allem bei Kindern (Aschenbecher, Zigarettenwasser) und bei Selbstmordversuchen erreicht werden, kommt es in einem sehr kurzen Zeitraum zum Kreislaufkollaps mit tödlicher Atemlähmung.

Kautabak führt aus zweierlei Gründen nicht zu akut gefährlichen Vergiftungen. Zum einen ist der Nikotingehalt im Kautabak geringer als in

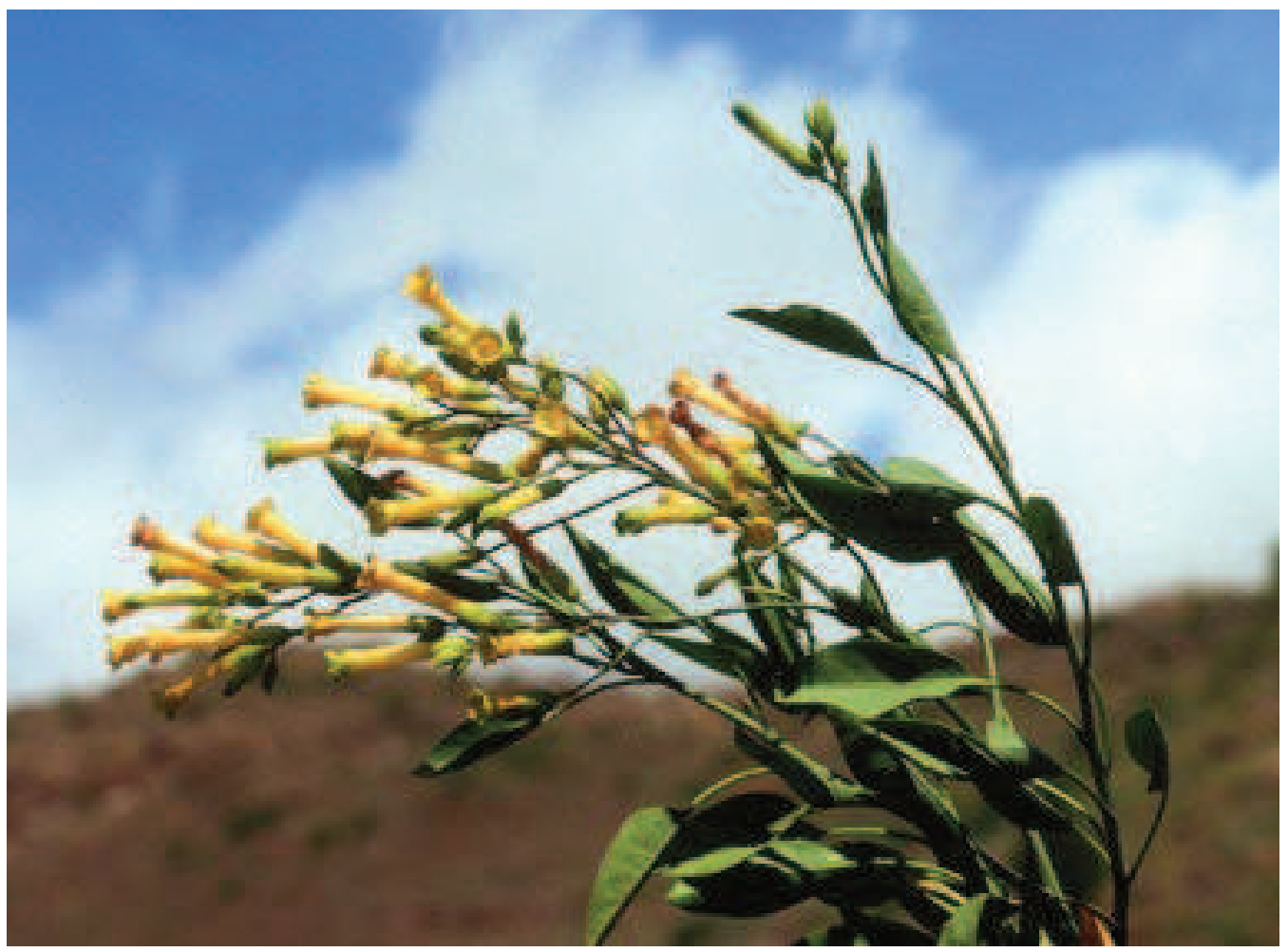


Rauchwaren, zum anderen wird ein Teil des $\mathrm{Ni}$ kotins in der Leber entgiftet, so dass nur ein geringer Teil in den Blutkreislauf gelangt. Auch beim Rauchen wird nur ein geringer Teil des im Tabak enthaltenen Nikotins aufgenommen. Dieser Anteil gelangt jedoch unmittelbar ins Blut. Dass das Rauchen von Tabak schädlich und insbesondere krebserregend sein kann, ist hinlänglich bekannt. Hierauf wird in Deutschland seit geraumer Zeit in einfallsreicher Vielfalt auf den Packungen hingewiesen, und es wird darüber hinaus auch immer mal wieder darüber diskutiert, dies durch abschreckende Bilder noch zu veranschaulichen, wie es in anderen Ländern schon praktiziert wird. Nikotin ist auch ein häufiger Bestandteil von Insektiziden und kann hier ebenfalls zu einer Gefahr für den Menschen werden. Früher nutzten Tabakbauern Tabakbrühe als Schädlingsbekämpfungsmittel (Kontaktinsektizid), die durch Abkochen der Blätter minderwertiger Sorten, von Tabakabfällen oder von besonders nikotinreichen Sorten gewonnen wurde.

\section{Tabak als Zierpflanze}

Als Zierpflanzen spielen die beiden bisher genannten Arten keine Rolle, wohl aber andere, die zum einjährigen Sommerblumensortiment gehören und einen angenehmen Duft verströmen wie z. B. der Berg- oder Duft-Tabak (N. sylvestris, Abb. 15), der Flügel-Tabak ( $N$. alata) und besonders die Sorten von Sanders Tabak (N. x sanderae $=N$. alata $\times$ forgetiana $)$, auch Niederer Zier-Tabak genannt. Die ursprünglichen Arten blühen nachts und werden von langrüsseligen Nachtfaltern bestäubt, die heutigen Gartenzüchtungen duften und blühen dagegen überwiegend auch tagsüber. Im Mittelmeergebiet und auf den Kanaren wird häufig der in Deutschland nicht winterharte, aus dem Südosten Süd-Amerikas stammende Blaugrüne Tabak gepflanzt, der dort stellenweise auch verwildert und sich einbürgert.

Abb. 16 (S. 64): Nicotiana glauca.

Abb. 17: Weißes Beet im Sommerflor 2009 des Palmengartens; in der Mitte Ziertabak.

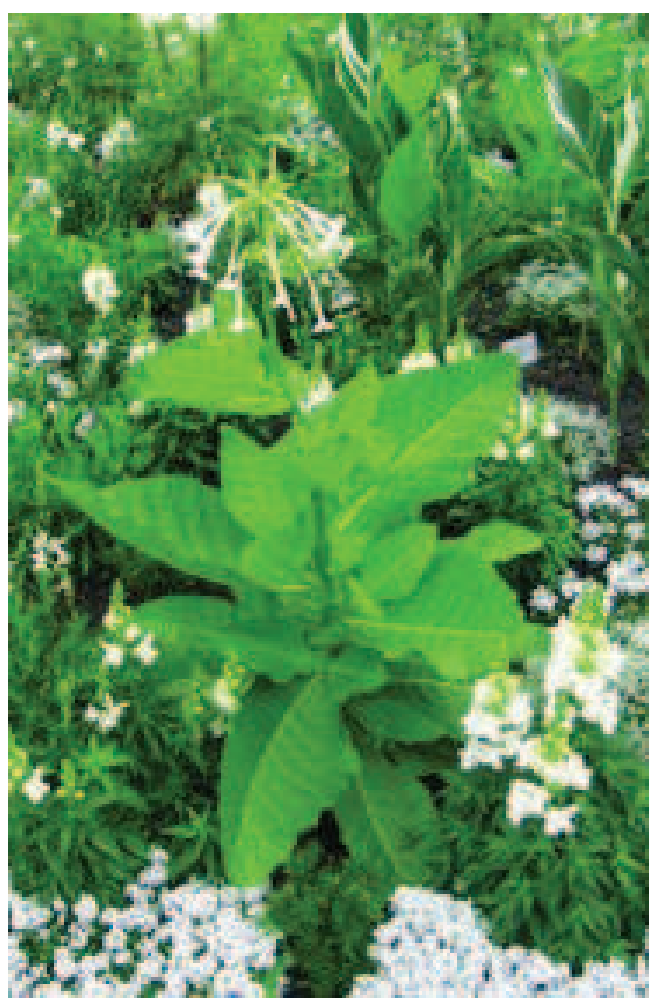

\section{Literatur}

Hammer, K. 2000: Solanales. - In: Urania-Pflanzenreich. Bd. 2. - Berlin.

Hiller, K. \& Melzig, M. F. 2003: Lexikon der Arzneipflanzen und Drogen. Bd. 2 (L bis Z). - Berlin, Heidelberg. LiEBEREI, R. \& REISDORF, C. 2007: Nutzpflanzenkunde.

Begründet von W. FranKe. 7. Aufl. - Stuttgart. RoTh, L., DAunderer, M. \& Kormann, K. 2008: Giftpflanzen, Pflanzengifte. 5. Aufl. - Hamburg. WOLTERS, B. 1994: Drogen, Pfeilgift und Indianermedizin. Arzneipflanzen aus Südamerika. - Greifenberg. Schmidbauer, W. \& Scheidt, J. vom 1999: Handbuch der Rauschdrogen. - Frankfurt am Main.

\section{Internetseite}

WIKIPEDIA: http://de.wikipedia.org/wiki/Tabak

(abgerufen am 18.02.2009) 